\title{
Sex Differences in Midbrain Dopamine D2-Type Receptor Availability and Association with Nicotine Dependence
}

\author{
Kyoji Okita ${ }^{1,2}$, Nicole Petersen', Chelsea L Robertson ${ }^{2,3}$, Andy C Dean ${ }^{1,4}$, Mark A Mandelkern ${ }^{2,5}$ and \\ Edythe D London $*, 1,2,3,4$
}

'Department of Psychiatry and Biobehavioral Sciences, David Geffen School of Medicine at the University of California Los Angeles, Los Angeles, CA, USA; ${ }^{2}$ Department of Research, VA Greater Los Angeles Healthcare System, Los Angeles, CA, USA; ${ }^{3}$ Department Molecular and Medical Pharmacology, David Geffen School of Medicine at the University of California Los Angeles, Los Angeles, CA, USA; ${ }^{4}$ The Brain Research Institute, David Geffen School of Medicine at the University of California Los Angeles, Los Angeles, CA, USA; ${ }^{5}$ Department of Physics, University of California Irvine, Irvine, CA, USA

\begin{abstract}
Women differ from men in smoking-related behaviors, among them a greater difficulty in quitting smoking. Unlike female smokers, male smokers have lower striatal dopamine D2-type receptor availability (binding potential, BPND) than nonsmokers and exhibit greater smoking-induced striatal dopamine release. Because dopamine D2-type autoreceptors in the midbrain influence striatal dopamine release, a function that has been linked to addiction, we tested for sex differences in midbrain dopamine D2-type receptor BPND and in relationships between midbrain BPND, nicotine dependence and striatal dopamine D2-type receptor BPND. Positron emission tomography was used with $\left[{ }^{18} \mathrm{~F}\right]$ fallypride to measure BPND in a midbrain region, encompassing the substantia nigra and ventral tegmental area, in 18 daily smokers (7 women, II men) and 19 nonsmokers (I0 women, 9 men). A significant sex-by-group interaction reflected greater midbrain BPND in female but not male smokers than in corresponding nonsmokers $\left(F_{1,32}=5.089, p=0.03\right)$. Midbrain BPND was positively correlated with BPND in the caudate nucleus and putamen in nonsmokers and female smokers but not in male smokers and with nicotine dependence in female but not in male smokers. Striatal BPND was correlated negatively with nicotine dependence and smoking exposure. These findings extend observations on dopamine D2-type receptors in smokers and suggest a sex difference in how midbrain dopamine D2-type autoreceptors influence nicotine dependence.

Neuropsychopharmacology (2016) 41, 2913-2919; doi:10.1038/npp.2016.105; published online I3 July 2016
\end{abstract}

\section{INTRODUCTION}

Cigarette smoking is the leading preventable cause of premature death in the United States and has been causally linked to diseases of nearly all organs in the body, diminished health, and adverse effects during fetal development (National Center for Chronic Disease, 2014). Nicotine, an essential component of tobacco products that promotes their compulsive use (Stolerman and Jarvis, 1995), interacts with nicotinic acetylcholine receptors in the brain, mimicking the action of acetylcholine and enhancing dopamine release and neurotransmission in pathways that mediate reward (for reviews, see Balfour, 2015; Cosgrove et al, 2015; Subramaniyan and Dani, 2015). Sex differences are seen in responses to nicotine with respect to reinforcement and reward in humans (Perkins, 2009) and nicotine selfadministration by animals (Jensen et al, 2015) and in the

\footnotetext{
*Correspondence: Dr ED London, Semel Institute for Neuroscience and Human Behavior, University of California Los Angeles, 740 Westwood Plaza, PO Box 175919, Los Angeles, CA 90024, USA, Tel: +(3I0) 825-0606 (voice), Fax: +(3I0) 825-08I2,

E-mail: elondon@mednet.ucla.edu

Received 25 February 2016; revised 7 June 2016; accepted 10 June 2016; accepted article preview online 22 June 2016
}

ability of smokers to quit (Pogun and Yararbas, 2009). Moreover, there are sex differences in the success of smoking-cessation therapy, with female smokers being less likely than males to achieve long-term abstinence (Cepeda-Benito et al, 2004; Perkins and Scott, 2008; Rose and Behm, 2014). To some extent, these findings may reflect sex differences in the regulation of central dopaminergic neurotransmission (Becker, 1999; Pilotte et al, 1984).

Sex differences have also been observed in studies of the effects of nicotine on cerebral glucose metabolism, where application of the nicotine patch reduced metabolic activity in women to eliminate sex differences observed in placebo conditions (Fallon et al, 2005). In addition, dopamine D2-type receptor availability, measured as binding potential (BPND), is lower in the caudate nucleus and putamen of male smokers as compared with nonsmokers (Fehr et al, 2008), but a parallel difference is absent in females (Brown et al, 2012). Sex differences in the time course of smoking-induced dopamine release have also been reported, with women responding consistently in the ventral striatum and women responding faster than men in the dorsal putamen (Cosgrove et al, 2014). Finally, women show faster nicotine accumulation in the brain when smoking than men, and menthol 
accelerates brain nicotine accumulation in men but not in women (Zuo et al, 2015).

Given that smoking-induced dopamine release differs between men and women, it is reasonable to consider the possibility of sex differences in the substantia nigra and ventral tegmental area (SN-VTA) within the midbrain (Gardner and Ashby, 2000), where striatal dopaminergic neurons originate. In the SN-VTA, dopamine D2-type receptors are predominantly somatodendritic autoreceptors, which inhibit dopamine release in terminal fields of the dopaminergic neurons (Khan et al, 1998; Sesack et al, 1994). Evidence of this type of regulation is seen in the negative correlation of dopamine D2-type receptor BPND in the SN-VTA (midbrain BPND) with a measure of striatal dopamine release following amphetamine administration (Buckholtz et al, 2010). Given the aforementioned sex differences in dopamine D2-type receptor BPND in the caudate nucleus and putamen (striatal BPND) and smoking behavior, we expected sex differences in midbrain BPND that may differ by smoking status. Further, we hypothesized that midbrain BPND is negatively related to nicotine dependence and influences the association of cigarette smoking with striatal BPND.

Positron emission tomography (PET) was performed using $\left[{ }^{18} \mathrm{~F}\right]$ fallypride, a radiotracer with high affinity for D2-type receptors (Mukherjee et al, 2002). In addition to the primary assessment of the interaction between sex and smoking status on midbrain BPND, relationships of midbrain BPND with striatal BPND, where smokers exhibit lower BPND than nonsmokers (Brown et al, 2012; Fehr et al, 2008), and with nicotine dependence were tested.

\section{MATERIALS AND METHODS}

\section{Participants}

All procedures were approved by the University of California Los Angeles Office for the Protection of Research Subjects. The participants were 18-55 years of age and were either daily smokers (5-20 cigarettes per day; 11 men and 7 women) or nonsmokers ( $<5$ cigarettes in their lifetimes; 10 men and 9 women). Participants were recruited through internet and local newspaper advertisements, and the participants were compensated for their time.

After receiving a complete explanation of the study procedures, each participant provided written, informed consent. Exclusion criteria (smokers and nonsmokers) were as follows: use of any psychotropic medication or substance except for nicotine and caffeine (in beverages) or meeting DSM-IV criteria for current or lifetime abuse of either alcohol or marijuana; central nervous system, cardiovascular, pulmonary, hepatic or systemic disease; human immunodeficiency virus seropositive status; pregnancy; lack of English fluency; MRI contraindications; or a current DSM-IV Axis-I diagnosis other than nicotine dependence, measured using the Structured Clinical Interview for DSM-IV. All participants were deemed physically healthy, on the basis of history and medical examination, and were right-handed, as indicated by the Edinburgh Handedness Questionnaire.

\section{Demographics and Smoking-Related Ratings}

The participants were characterized with regard to age and sex, as well as features of smoking exposure, including

number of cigarettes smoked per day, years of smoking, pack-years, and nicotine dependence, as measured by the Fagerström Test for Nicotine Dependence (FTND). Information was also obtained regarding other substances used in the past month (eg, marijuana, alcohol), and abstinence was verified by urine toxicology at intake and immediately before the PET scans. Exhaled carbon monoxide (CO) was measured using a Micro $^{+}$Smokerlyzer (Bedfont Scientific, Kent, UK) to verify smoker or nonsmoker status at intake.

\section{PET and MRI Scanning}

Participants were instructed to refrain from smoking in the $2 \mathrm{~h}$ before PET scanning, which was performed with $\left[{ }^{18} \mathrm{~F}\right]$ fallypride. Data were acquired using a Siemens ECAT EXACT HR+ scanner, which has an in-plane resolution full-width at half-maximum (FWHM) of $4.6 \mathrm{~mm}$, axial resolution FWHM of $3.5 \mathrm{~mm}$, and an axial field of view of $15.52 \mathrm{~cm}$ in the three-dimensional scanning mode. A transmission scan was conducted using a rotating ${ }^{68} \mathrm{Ga} /{ }^{68} \mathrm{Ge}$ rod source for attenuation correction. Images were reconstructed using the ECAT v7.3 Ordered Subset Expectation Maximization method (6 iterations, 16 subsets) with a Gaussian postreconstruction filter for each 1-min frames.

Participants were placed in the supine position for scanning, with the head positioned and secured with plastic tape to avoid movement during the scan. Emission data were collected for $80 \mathrm{~min}$ after the intravenous injection of $\left[{ }^{18} \mathrm{~F}\right]$ fallypride $(5.14 \pm 0.30 \mathrm{mCi}$, specific activity $6.89 \pm 5.23$ $\mathrm{Ci} / \mu \mathrm{mol})$. Participants were then removed from the scanner for a 20-min break after which they returned to the scanner and were repositioned. Emission data were then collected for a second 80 -min period.

Structural MRI scans of the brain were acquired on a $1.5 \mathrm{~T}$ Siemens Sonata tomograph for co-registration with PET images and definition of volumes of interest (VOIs; see below for details). A high-resolution, sagittal, T1-weighted, 3-dimensional, volumetric scan was acquired using a wholebrain magnetization-prepared rapid acquisition with gradient echo (MPRAGE) sequence $(\mathrm{TR}=1900 \mathrm{~ms}, \mathrm{TE}=4.38 \mathrm{~ms}$, flip angle $=15$, field of view $=256 \times 256 \times 160$, 160 slices, thickness $=1 \mathrm{~mm}$ ).

\section{PET Data Processing}

Image processing and VOIs. Reconstructed PET data consisting of 1601 -min frames were combined into 16 images, each containing data averaged over $10 \mathrm{~min}$. FSL MCFLIRT (FMRIB Centre, Department of Clinical Neurology, University of Oxford, Oxford, UK) was used to correct for head motion (Jenkinson et al, 2002). The images were then co-registered to the MPRAGE image using a six-parameter, rigid-body spatial transformation (FSL FLIRT).

The primary question of interest, regarding the potential interaction between sex and smoking status on midbrain BPND, was addressed using a midline SN-VTA VOI (see Figure 1). Because of its small size and variability in positioning, the SN-VTA VOI was drawn using a data-driven approach according to a published procedure (Zald et al, 2010). An average PET image was created using all co-registered 10-min frames of emission data acquired. Regions containing the SN-VTA were hand-drawn on six 


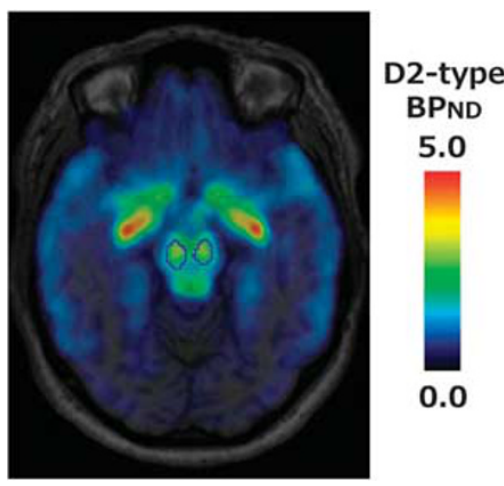

Figure I SN-VTA VOI in an individual subject. Volume of interest (VOI) in the midbrain, encompassing the substantia nigra and ventral tegmental area (SN-VTA), is shown with a blue line on the fusion image of the structural MRI and the binding potential (BPND) map of a single subject.

successive $z$-axis slices (1-mm thickness) in MNI152 space. The resulting VOI was transformed to native space using FSL FNIRT. The voxels of this VOI were sequentially selected in order of signal intensity, starting from the voxel with the maximum signal intensity until the volume of selected voxels reached 756 voxels, each of dimensions $1 \mathrm{~mm}^{3}$ to form the final SN-VTA VOI.

Striatal VOIs were also created for modeling of the PET data and for further investigation as described below. VOIs of the caudate nucleus and putamen were derived from individual MPRAGE images using autosegmentation procedures in FSL FIRST (Patenaude et al, 2011). The cerebellum, which has a very low level of specific binding for $\left[{ }^{18} \mathrm{~F}\right]$ fallypride (Mukherjee et al, 2002), was used as the reference region. VOIs were manually created for the right and left cerebellar hemispheres in MNI152 space, combined into a single VOI, and transformed into native space with FSL FNIRT.

Modeling of PET data. Time-activity data within VOIs were extracted from motion-corrected, co-registered PET images and imported into PMOD Kinetic Modeling (PKIN) (PMOD Technologies, Zurich, Switzerland). The simplified reference tissue model (SRTM) (Lammertsma and Hume, 1996) was used to estimate $\mathrm{k} 2$ ', the rate parameter for the transfer of the radiotracer from the reference-region tissue compartment to the plasma, for the striatal VOIs.

The VOI time-activity curves were refit using the SRTM2 model (Wu and Carson, 2002) and PKIN, holding fixed the $\mathrm{k} 2$ ' value derived from the striatal VOIs using SRTM. BPND, an index of receptor availability, was then calculated as $\mathrm{BPND}=\mathrm{R} 1^{*} \mathrm{k} 2^{\prime} / \mathrm{k} 2 \mathrm{a}-1$, where $\mathrm{R} 1=\mathrm{K} 1 / \mathrm{K} 1^{\prime}$ is the ratio of tracer-delivery parameters from plasma to tissues in the target region and reference region, and $\mathrm{k} 2 \mathrm{a}$ is the single-compartment rate parameter for transfer from the target-region tissue compartment to plasma.

\section{Statistical Analyses}

Differences in demographic data between groups (nonsmokers, smokers) and in smoking-related measures, including FTND, pack-years, and number of cigarettes per day, between males and females in the smoker group were evaluated by unpaired Student's $t$-tests.
ANCOVA was used to evaluate the main effects of sex and subject group (nonsmokers $v s$ smokers), as well as the interaction between sex and group on midbrain BPND. Age was included in the model as a covariate of no interest, chosen beforehand in consideration of evidence for age-related decline in dopamine-receptor density in the brain (Kaasinen et al, 2000). After identifying a sex-by-group interaction effect, separate ANCOVAs were performed for each group with sex as the single factor and age as a covariate of no interest.

In order to avoid finding an artefactual correlation owing to variation in tracer dose, correlations of midbrain BPND with caudate nucleus and with putamen BPND were tested controlling for whole-brain BPND, evaluated from all voxels with $\mathrm{BPND} \geqslant 1.0$ (see Supplementary Materials and Methods for the voxel-wise analysis). Relationships of BPND in the midbrain, caudate nucleus, and putamen with smokingrelated measures (ie, nicotine dependence, cigarettes per day) were evaluated using partial correlation analysis, controlling for age. In analyses involving pack-years, however, age was not controlled for because it was expected to be collinear with pack-years. Correlation coefficient comparisons were performed using Fisher's $r$ to $z$ transformation.

These statistical analyses were conducted using SPSS IBM 19 (IBM, Armonk, NY, USA). The criterion for statistical significance was $p<0.05$, two-tailed. Results are shown as mean \pm SD

\section{RESULTS}

\section{Participant Characteristics}

Participant characteristics are shown in Table 1. The groups did not differ in age although there was a trend for the smokers (39.1 \pm 9.46 (men), $37.9 \pm 8.65$ (women)) to be older than the nonsmokers $(36.2 \pm 8.44$ (men), $31.6 \pm 9.72$ (women) $(p=0.13)$. Smokers exhibited significantly higher CO levels in expired air than nonsmokers during screening for inclusion in the study $(p<0.001)$, with no sex difference (20.6 \pm 10.19 p.p.m. (men), $14.4 \pm 9.29$ p.p.m. (women); $p=0.22$ ). Smokers also showed moderate nicotine dependence as indicated by FTND scores, with no sex difference (4.2 \pm 2.23 (men), $3.4 \pm 2.07$ (women); $p=0.48$ ). There were also no sex differences in smoking exposure, as indicated by pack-years (15.2 \pm 11.49 (men), $11.7 \pm 7.67$ (women); $p=0.49)$ and in number of cigarettes smoked per day $(14.0 \pm 4.63$ (men), $12.3 \pm 3.04$ (women); $p=0.40)$. Thirteen smokers met the criteria for current nicotine dependence, whereas none met criteria for any lifetime dependence on other substances, as defined in DSM-IV. Twenty participants (six male smokers, four female smokers, five male and female nonsmokers) reported having used alcohol in the month before study, endorsing alcohol use on $4.2 \pm 3.62$ days. Two male smokers reported having used marijuana, each reporting use on only 1 day in the month before study.

\section{Interaction of Sex with Smoking Status on Midbrain Dopamine D2-Type BPND}

There was a significant interaction between sex and group (smoker, nonsmoker) on midbrain BPND $\left(\mathrm{F}_{1,32}=5.089\right.$, $p=0.03$ ), but no significant main effects of sex or group (sex: $F_{1,32}=1.156, p=0.29$; group: $F_{1,32}=0.962, p=0.33$ ). 
Table I Characteristics of Research Participants

\begin{tabular}{|c|c|c|c|c|c|c|}
\hline & \multicolumn{4}{|c|}{ Participant group } & \multirow{3}{*}{$\begin{array}{c}\text { Group } \\
\text { difference } \\
\text { p-Value }\end{array}$} & \multirow{3}{*}{$\begin{array}{c}\text { Sex difference: } \\
\text { nonsmoker/smoker } \\
\text { p-Value }\end{array}$} \\
\hline & \multicolumn{2}{|c|}{ Nonsmoker $(n=19)$} & \multicolumn{2}{|c|}{ Smoker $(n=18)$} & & \\
\hline & Men $(n=10)$ & Women $(n=9)$ & Men $(n=I I)$ & Women $(n=7)$ & & \\
\hline Age (years) & $36.2 \pm 8.44$ & $31.6 \pm 9.72$ & $39.1 \pm 9.46$ & $37.9 \pm 8.65$ & 0.13 & $0.28 / 0.78$ \\
\hline Pack-years & NA & NA & $15.2 \pm 11.5$ & $11.7 \pm 7.67$ & NA & 0.49 \\
\hline Number of cigarettes/day & NA & NA & $14.0 \pm 4.63$ & $12.3 \pm 3.04$ & NA & 0.40 \\
\hline
\end{tabular}

Abbreviations: CO, carbon monoxide; FTND, Fagerström Test for Nicotine Dependence.

Values shown are means \pm s.d. Differences by group and sex were evaluated using unpaired Student's $t$-test.

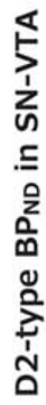

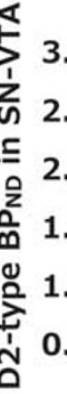
3.0
2.5
2.0
1.5
1.0
0.5

\section{.8000}

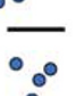

$\therefore$

(

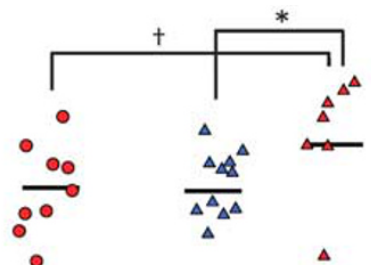

Nonsmoker

Smoker

Figure 2 Interaction of sex and smoking status on midbrain D2-type BPND. Plots of individual values of midbrain BPND by sex and group. Horizontal lines show the mean value of each cohort. Interaction effect between sex and smoking status was observed by ANCOVA $\left(F_{1,32}=5.089\right.$, $p=0.03)$. Post-hoc analyses revealed significantly higher BPND in female than in male smokers $\left.{ }^{*} p=0.03\right)$, and a trend for higher BPND in female smokers than in nonsmokers $\left({ }^{\dagger} p=0.09\right)$. BPND values are adjusted for age. Blue circles: male nonsmokers; red circles: female nonsmokers; Blue triangles: male smokers; red triangles: female smokers.

Post-hoc analyses revealed that female smokers had higher midbrain BPND than male smokers $(1.74 \pm 0.29$ (men), $2.18 \pm 0.49$ (women); $\mathrm{F}_{1,15}=5.482, p=0.03$ ), but there was no sex difference in midbrain BPND among nonsmokers $\left(1.93 \pm 0.38\right.$ (men), $1.83 \pm 0.42 \quad$ (women); $\mathrm{F}_{1,16}=0.689$, $p=0.42$ ). There also was a trend toward higher midbrain BPND in female smokers than in female nonsmokers $\left(\mathrm{F}_{1,13}=3.290, \quad p=0.09\right)$, whereas there was no group difference in men $\left(F_{1,18}=1.215, p=0.29\right)$ (Figure 2).

\section{Relationship of Midbrain BPND with Nicotine Dependence}

Midbrain BPND was negatively correlated with FTND in the sample of all smokers $(r=-0.591, p=0.01)$; post-hoc analysis revealed that this effect reflected a significant negative correlation of midbrain BPND with FTND in female smokers $(r=-0.989, p<0.001)$ but not in male smokers $(r=-0.425$, $p=0.22$ ) (Figure 3 ). The correlation coefficients differed significantly by $\operatorname{sex}(z=3.50, p<0.001)$. Other smokingrelated measures, such as pack-years $(r=-0.304, p=0.22)$

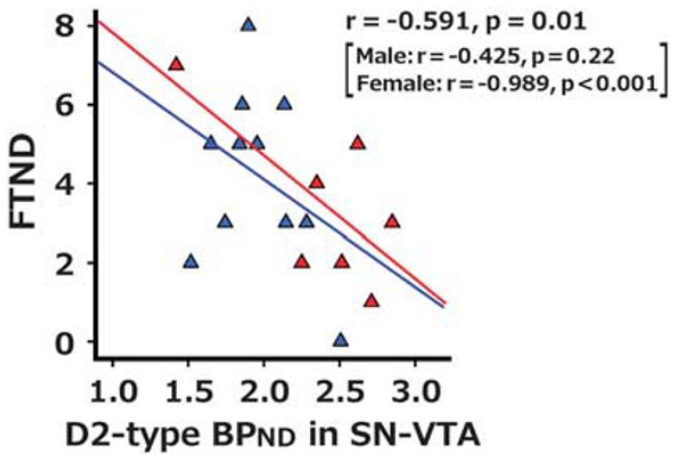

Figure 3 Relationship of midbrain D2-type BPND with nicotine dependence. Midbrain BPND was negatively correlated with FTND in the sample of all smokers $(r=-0.59 \mathrm{I}, p=0.0 \mathrm{I})$. Post-hoc analysis in each sex showed significant correlation in female smokers $(r=-0.989, p<0.00 \mathrm{I})$ but not in male smokers $(r=-0.425, p=0.22)$. The correlation coefficients differed by $\operatorname{sex}(z=3.50, p<0.00 \mathrm{I})$.

and number of cigarettes smoked per day $(r=-0.284$, $p=0.27$ ), were not significantly related to midbrain BPND.

\section{Relationships of Striatal BPND, Midbrain BPND, Nicotine Dependence, and Smoking Exposure}

$\mathrm{BPND}$ values in both the caudate nucleus and putamen were positively correlated with midbrain BPND in both nonsmokers and smokers of each sex (male nonsmokers: $r$ 's $=0.752$ and 0.729; female nonsmokers: $r^{\prime} s=0.893$ and 0.816 ; female smokers: $r$ 's $=0.776$ and 0.813 ; all $p$ 's $<0.05$ uncorrected for multiple comparisons), except for male smokers ( $r$ 's $=-0.132$ and $0.041, p$ 's $>0.5$ uncorrected for multiple comparisons). FTND was significantly negatively correlated with striatal BPND (caudate: $r=-0.556, p=0.02$; putamen: $r=-0.587$, $p=0.01$ ). Although correlations between striatal BPND and pack-years did not reach significance in the combined sample (caudate: $r=-0.378, p=0.12$; putamen: $r=-0.414, p=0.09$ ), the correlations between pack-years and striatal BPND in men (caudate: $r=-0.732, p=0.01$; putamen: $r=-0.727, p=0.01$ ) but not in women (caudate: $r=-0.186, p=0.69$; putamen: $r=-0.073, p=88$ ) were significant. 


\section{DISCUSSION}

The central findings of this study concern midbrain dopamine D2-type receptors and their potential role in sex differences in striatal dopamine signaling in smokers and in clinical features of nicotine dependence. A significant interaction between sex and smoking status on midbrain BPND was observed, reflecting higher values in female smokers than in male smokers, and a trend for midbrain BPND to be higher in female smokers than in female nonsmokers but not in male smokers.

Previous studies have consistently reported lower D2-type receptor availability in the striata of individuals with substance-use disorders compared with healthy subjects, and animal studies have suggested that chronic drug exposure contributes to impairment in the dopaminergic system (see Trifilieff and Martinez, 2014; Volkow et al, 2011 for reviews). Notably, in rats, chronic nicotine administration using an implanted pump for 14 days produces downregulation of D2receptor density in the basal ganglia, preferentially in the ventral striatum (Janson et al, 1992), and counteracts lesioninduced upregulation of D2-receptor density in the striatum. These effects have been attributed to nicotine-induced dopamine release (Janson et al, 1994). Another study demonstrated increased D3-receptor density in the shell of the nucleus accumbens but no significant alterations in D2receptor density owing to nicotine administration for 5 days in naive rats (Le Foll et al, 2003). However, these studies differed in the nicotine administration regimens used. Yet another study suggested that at least five administrations of nicotine are required to evoke dopamine release in naive rats (Cadoni and Di Chiara, 2000).

Dopamine D2-type receptors in SN-VTA are predominantly of the D2S isoform and, as such, have autoinhibitory effects on neuronal excitability and thereby on dopamine release in terminal fields (Beckstead et al, 2004; Rice and Patel, 2015). Thus greater midbrain BPND in female smokers could lead to lower smoking-induced dopamine release and therefore less downregulation of striatal D2-type receptors. Lower striatal D2-type BPND in male but not in female smokers, as reported previously (Brown et al, 2012), may then be paired with higher midbrain BPND in female but not in male smokers. The view is supported by the significant negative correlation of striatal BPND with smoking exposure in men but not in women. Moreover, a negative modulatory role of midbrain D2-type autoreceptors on striatal dopamine release is consistent with the positive correlation of midbrain BPND with striatal BPND in nonsmokers of both sexes and female smokers but not in male smokers. Thus higher midbrain D2-type BPND in female but not male smokers would limit striatal dopamine release and potentially resultant downregulation of striatal D2-type BPND owing to chronic nicotine exposure.

Whereas female smokers exhibited higher midbrain BPND than female nonsmokers, they also showed a negative correlation of midbrain BPND with nicotine dependence. The latter observation suggests that high densities of midbrain D2 autoreceptors in women may protect against nicotine dependence by preventing downregulation of D2-type receptors in the striatum. Supporting the view that midbrain autoreceptors protect against drug addiction are studies of mice, in which D2-autoreceptor deletion produced hypersensitivity to cocaine (Bello et al, 2011), enhanced cocaine use, and increased dopamine release in the nucleus accumbens
(Holroyd et al, 2015). Because smoking-induced striatal dopamine release is related to relief of cigarette craving and withdrawal symptoms during abstinence (Brody et al, 2004, 2006; Le Foll et al, 2014), lower midbrain BPND and greater associated smoking-induced dopamine release may be associated with greater negative reinforcement from smoking in men than in women. This effect may confer greater nicotine dependence, as supported by the observation that the magnitude of dopamine release following the use of nicotine gum is positively correlated with nicotine dependence in male smokers (Takahashi et al, 2008).

This study is not without limitations, one of which is relatively small number of participants, necessitating the replication of these findings in a larger sample to demonstrate its reproducibility. Another limitation is that menstrual cycle phases of the female participants during testing were not recorded, and this uncertainty may have introduced unexplained variance into the measurements. A trend toward higher binding rate constant $k_{3}$ to the D2-type receptor in the caudate nucleus in the luteal phase of the menstrual cycle in healthy women (Wong et al, 1988) and higher striatal D2-type BPND during the luteal phase compared with the follicular phase in cynomolgus monkeys (Czoty et al, 2009) have been reported. Whereas one study of human subjects indicated lower D2-type BPND in posterior putamen in the luteal phase than the follicular phase (Munro et al, 2006), another showed no change in putamen D2-type receptor binding with menstrual-cycle phase (Nordstrom et al, 1998). Although these results are inconsistent, controlling menstrual-cycle phase in studies of D2-type BPND is recommended for future studies. Furthermore, sex hormones can influence nicotine metabolism, and in general women metabolize nicotine faster than men (Benowitz et al, 2006). This difference, along with other factors that may affect nicotine metabolism, may have global effects on nicotine exposure and D2-type BPND.

Other limitations reflect those associated with the PET measurement. Participants were studied when abstinent from smoking for at least $2 \mathrm{~h}$ but were not required to maintain longer abstinence so as to minimize discomfort from abstinence during the PET procedure, which took $180 \mathrm{~min}$. Because the plasma half-life of nicotine after intravenous infusion or cigarette smoking is approximately $2 \mathrm{~h}$ (Benowitz et al, 2009), residual nicotine was likely to have been present. Smoking promotes prompt striatal dopamine release, when measured using $\left[{ }^{11} \mathrm{C}\right]$ raclopride within a bolusplus-infusion paradigm, presumably in part owing to an acute effect of nicotine (Brody et al, 2004; Cosgrove et al, 2014). However, no difference in BPND of any brain region was measured using $\left[{ }^{18} \mathrm{~F}\right]$ fallypride when comparing data obtained $15 \mathrm{~min}$ after smoking with data after $24 \mathrm{~h}$ of abstinence (Fehr et al, 2008). This result may be a consequence of the extended (3-h) duration of a fallypride scan that was initiated after smoking was completed. It suggests that BPND measured this way using $\left[{ }^{18} \mathrm{~F}\right]$ fallypride is not sensitive to tonic intrasynaptic dopamine levels as altered by recent smoking and that any potential confound in this study from recent smoking was minimal. That dopamine depletion with $\alpha$-methyltyrosine has no effect on $\left[{ }^{18} \mathrm{~F}\right]$ fallypride BPND is further evidence that $\left[{ }^{18} \mathrm{~F}\right]$ fallypride BPND is minimally affected by individual differences in tonic intrasynaptic dopamine level (Cropley et al, 2008). Nevertheless, in future studies, recording $\mathrm{CO}$ in exhaled air and the time stamp of 
smoking, as well as nicotine and related metabolite levels in blood at the time of scanning, is strongly recommended to address this potentially confounding effect.

Evidence that up to $20 \%$ of $\left[{ }^{18} \mathrm{~F}\right]$ fallypride may be bound to D3 dopamine receptors in vivo (Mukherjee et al, 2015) and that both D2 and D3 autoreceptors are present in the SN-VTA (Gurevich and Joyce, 1999) precludes making definitive statements regarding either receptor subtype. Moreover, autoradiographic demonstration of greater density of D3 than D2 dopamine receptors in the aged human brain (Sun et al, 2012) suggests that the greater SN-VTA BPND seen in female smokers may reflect a greater effect on D3 than D2 receptors. Studies using D3-receptor selective agents support the existence of a mechanism for control of dopamine release by D3 autoreceptors (for a review, see Sokoloff et al, 2006), although they may have only a minor functional role (Davila et al, 2003). Finally, a causal relationship between smoking and group differences in midbrain BPND in females cannot be claimed. To do so would have required PET assay before the participants initiated smoking. Also, future studies might test for sex differences in midbrain BPND and in smoking-induced dopamine release in the same subject sample.

Despite these limitations, the new finding of a sex difference in midbrain D2-type receptors in smokers may help explain some sex differences in smoking behavior. As described above, higher midbrain BPND in female $v s$ male smokers may protect striatal D2-type receptors from the effects of smoking and may be to linked to less severe nicotine dependence. Further, this difference may help explain why female smokers derive less benefit from nicotine replacement therapy than male smokers (Perkins and Scott, 2008). It has been suggested that sex differences in nicotine replacement therapy may be due to a nicotine-induced increase in $\beta 2^{*}$ nicotinic acetylcholine receptor availability in males but not in females, as shown in human as well as rat studies (Cosgrove et al, 2012; Koylu et al, 1997). We suggest that, aside from differences in the direct action of nicotine at nicotinic acetylcholine receptors, female smokers may not respond as well as males because nicotineinduced dopamine release is limited by a higher density of midbrain D2 autoreceptors. Future investigations are warranted to link the sex differences observed here with sex differences in smoking behavior, in order to provide optimal treatments for smoking cessation.

\section{FUNDING AND DISCLOSURE}

This research was supported, in part, by grants from the National Institute on Drug Abuse (R01 DA015179, R01 DA020726, P20 DA022539, EDL; K23 DA027734, ACD) and the National Center for Research Resources (M01 RR00865) and endowments from the Thomas P and Katherine K Pike Chair in Addiction Studies (EDL) and the Marjorie $M$ Greene Trust. KO was partly supported by the Department of Psychiatry, Graduate School of Medicine, Chiba University, DOMONKAI fund. CLR was supported by T32 DA024635. The authors declare no conflict of interest.

\section{ACKNOWLEDGMENTS}

We thank Bryan Garrison and Dmitriy Gekker for their assistance with this investigation.

\section{REFERENCES}

Balfour DJ (2015). The role of mesoaccumbens dopamine in nicotine dependence. Curr Top Behav Neurosci 24: 55-98.

Becker JB (1999). Gender differences in dopaminergic function in striatum and nucleus accumbens. Pharmacol Biochem Behav 64: 803-812.

Beckstead MJ, Grandy DK, Wickman K, Williams JT (2004). Vesicular dopamine release elicits an inhibitory postsynaptic current in midbrain dopamine neurons. Neuron 42: 939-946.

Bello EP, Mateo Y, Gelman DM, Noain D, Shin JH, Low MJ et al (2011). Cocaine supersensitivity and enhanced motivation for reward in mice lacking dopamine D2 autoreceptors. Nat Neurosci 14: 1033-1038.

Benowitz NL, Hukkanen J, Jacob P (2009). Nicotine chemistry, metabolism, kinetics and biomarkers. Handb Exp Pharmacol 192: 29-60.

Benowitz NL, Lessov-Schlaggar CN, Swan GE, Jacob P 3rd (2006). Female sex and oral contraceptive use accelerate nicotine metabolism. Clin Pharmacol Ther 79: 480-488.

Brody AL, Mandelkern MA, Olmstead RE, Scheibal D, Hahn E, Shiraga $S$ et al (2006). Gene variants of brain dopamine pathways and smoking-induced dopamine release in the ventral caudate/ nucleus accumbens. Arch Gen Psychiatry 63: 808-816.

Brody AL, Olmstead RE, London ED, Farahi J, Meyer JH, Grossman $P$ et al (2004). Smoking-induced ventral striatum dopamine release. Am J Psychiatry 161: 1211-1218.

Brown AK, Mandelkern MA, Farahi J, Robertson C, Ghahremani DG, Sumerel B et al (2012). Sex differences in striatal dopamine D2/D3 receptor availability in smokers and non-smokers. Int J Neuropsychopharmacol 15: 989-994.

Buckholtz JW, Treadway MT, Cowan RL, Woodward ND, Li R, Ansari MS et al (2010). Dopaminergic network differences in human impulsivity. Science 329: 532.

Cadoni C, Di Chiara G (2000). Differential changes in accumbens shell and core dopamine in behavioral sensitization to nicotine. Eur J Pharmacol 387: R23-R25.

Cepeda-Benito A, Reynoso JT, Erath S (2004). Meta-analysis of the efficacy of nicotine replacement therapy for smoking cessation: differences between men and women. J Consult Clin Psychol 72: $712-722$.

Cosgrove KP, Esterlis I, McKee SA, Bois F, Seibyl JP, Mazure CM et al (2012). Sex differences in availability of beta $2^{\star}$-nicotinic acetylcholine receptors in recently abstinent tobacco smokers. Arch Gen Psychiatry 69: 418-427.

Cosgrove KP, Esterlis I, Sandiego C, Petrulli R, Morris ED (2015). Imaging Tobacco Smoking with PET and SPECT. Curr Top Behav Neurosci 24: 1-17.

Cosgrove KP, Wang S, Kim SJ, McGovern E, Nabulsi N, Gao H et al (2014). Sex differences in the brain's dopamine signature of cigarette smoking. J Neurosci 34: 16851-16855.

Cropley VL, Innis RB, Nathan PJ, Brown AK, Sangare JL, Lerner A et al (2008). Small effect of dopamine release and no effect of dopamine depletion on $\left[{ }^{18} \mathrm{~F}\right]$ fallypride binding in healthy humans. Synapse 62: 399-408.

Czoty PW, Riddick NV, Gage HD, Sandridge M, Nader SH, Garg S et al (2009). Effect of menstrual cycle phase on dopamine D2 receptor availability in female cynomolgus monkeys. Neuropsychopharmacology 34: 548-554.

Davila V, Yan Z, Craciun LC, Logothetis D, Sulzer D (2003). D3 dopamine autoreceptors do not activate G-protein-gated inwardly rectifying potassium channel currents in substantia nigra dopamine neurons. J Neurosci 23: 5693-5697.

Fallon JH, Keator DB, Mbogori J, Taylor D, Potkin SG (2005). Gender: a major determinant of brain response to nicotine. Int $J$ Neuropsychopharmacol 8: 17-26.

Fehr C, Yakushev I, Hohmann N, Buchholz HG, Landvogt C, Deckers $\mathrm{H}$ et al (2008). Association of low striatal dopamine $\mathrm{d} 2$ 
receptor availability with nicotine dependence similar to that seen with other drugs of abuse. Am J Psychiatry 165: 507-514.

Gardner EL, Ashby CR Jr (2000). Heterogeneity of the mesotelencephalic dopamine fibers: physiology and pharmacology. Neurosci Biobehav Rev 24: 115-118.

Gurevich EV, Joyce JN (1999). Distribution of dopamine D3 receptor expressing neurons in the human forebrain: comparison with D2 receptor expressing neurons. Neuropsychopharmacology 20: $60-80$.

Holroyd KB, Adrover MF, Fuino RL, Bock R, Kaplan AR, Gremel CM et al (2015). Loss of feedback inhibition via D2 autoreceptors enhances acquisition of cocaine taking and reactivity to drugpaired cues. Neuropsychopharmacology 40: 1495-1509.

Janson AM, Hedlund PB, Fuxe K, von Euler G (1994). Chronic nicotine treatment counteracts dopamine $\mathrm{D} 2$ receptor upregulation induced by a partial meso-diencephalic hemitransection in the rat. Brain Res 655: 25-32.

Janson AM, Hedlund PB, Hillefors M, von Euler G (1992). Chronic nicotine treatment decreases dopamine $\mathrm{D} 2$ agonist binding in the rat basal ganglia. Neuroreport 3: 1117-1120.

Jenkinson M, Bannister P, Brady M, Smith S (2002). Improved optimization for the robust and accurate linear registration and motion correction of brain images. Neuroimage 17: 825-841.

Jensen KP, DeVito E, Valentine G, Gueorguieva R, Sofuoglu M (2015). IV Nicotine self-administration in smokers: dose-response function and sex differences. Neuropsychopharmacology 41: 2034-2040.

Kaasinen V, Vilkman H, Hietala J, Nagren K, Helenius H, Olsson H et al (2000). Age-related dopamine D2/D3 receptor loss in extrastriatal regions of the human brain. Neurobiol Aging 21: 683-688.

Khan ZU, Mrzljak L, Gutierrez A, de la Calle A, Goldman-Rakic PS (1998). Prominence of the dopamine D2 short isoform in dopaminergic pathways. Proc Natl Acad Sci USA 95: 7731-7736.

Koylu E, Demirgoren S, London ED, Pogun S (1997). Sex difference in up-regulation of nicotinic acetylcholine receptors in rat brain. Life Sci 61: 185-190.

Lammertsma AA, Hume SP (1996). Simplified reference tissue model for PET receptor studies. Neuroimage 4(Pt 1): 153-158.

Le Foll B, Diaz J, Sokoloff P (2003). Increased dopamine D3 receptor expression accompanying behavioral sensitization to nicotine in rats. Synapse 47: 176-183.

Le Foll B, Guranda M, Wilson AA, Houle S, Rusjan PM, Wing VC et al (2014). Elevation of dopamine induced by cigarette smoking: novel insights from a [11C]-+-PHNO PET study in humans. Neuropsychopharmacology 39: 415-424.

Mukherjee J, Christian BT, Dunigan KA, Shi B, Narayanan TK, Satter $\mathrm{M}$ et al (2002). Brain imaging of ${ }^{18} \mathrm{~F}$-fallypride in normal volunteers: blood analysis, distribution, test-retest studies, and preliminary assessment of sensitivity to aging effects on dopamine D-2/D-3 receptors. Synapse 46: 170-188.

Mukherjee J, Constantinescu CC, Hoiang AT, Jerjian T, Majji D, Pan ML (2015). Dopamine D3 receptor binding of (18)Ffallypride: evaluation using in vitro and in vivo PET imaging studies. Synapse 69: 577-591.

Munro CA, McCaul ME, Wong DF, Oswald LM, Zhou Y, Brasic J et al (2006). Sex differences in striatal dopamine release in healthy adults. Biol Psychiatry 59: 966-974.

National Center for Chronic Disease PHPOo, Smoking Health (2014). Reports of the Surgeon General. The Health Consequences of Smoking-50 Years of Progress: A Report of the Surgeon General. Centers for Disease Control and Prevention (US): Atlanta, GA, USA.

Nordstrom AL, Olsson H, Halldin C (1998). A PET study of D2 dopamine receptor density at different phases of the menstrual cycle. Psychiatry Res 83: 1-6.
Patenaude B, Smith SM, Kennedy DN, Jenkinson M (2011). A Bayesian model of shape and appearance for subcortical brain segmentation. Neuroimage 56: 907-922.

Perkins KA (2009). Sex differences in nicotine reinforcement and reward: influences on the persistence of tobacco smoking. Nebr Symp Motiv 55: 143-169.

Perkins KA, Scott J (2008). Sex differences in long-term smoking cessation rates due to nicotine patch. Nicotine Tob Res 10: $1245-1250$.

Pilotte NS, Burt DR, Barraclough CA (1984). Ovarian steroids modulate the release of dopamine into hypophysial portal blood and the density of anterior pituitary $[3 \mathrm{H}]$ spiperone-binding sites in ovariectomized rats. Endocrinology 114: 2306-2311.

Pogun S, Yararbas G (2009). Sex differences in nicotine action. Handb Exp Pharmacol 192: 261-291.

Rice ME, Patel JC (2015). Somatodendritic dopamine release: recent mechanistic insights. Philos Trans $R$ Soc Lond Ser B Biol Sci 370: 1672.

Rose JE, Behm FM (2014). Combination treatment with varenicline and bupropion in an adaptive smoking cessation paradigm. Am J Psychiatry 171: 1199-1205.

Sesack SR, Aoki C, Pickel VM (1994). Ultrastructural localization of D2 receptor-like immunoreactivity in midbrain dopamine neurons and their striatal targets. J Neurosci 14: 88-106.

Sokoloff P, Diaz J, Le Foll B, Guillin O, Leriche L, Bezard E et al (2006). The dopamine D3 receptor: a therapeutic target for the treatment of neuropsychiatric disorders. CNS Neurol Disord Drug Targets 5: 25-43.

Stolerman IP, Jarvis MJ (1995). The scientific case that nicotine is addictive. Psychopharmacology (Berl) 117: 2-10 discussion 14-20.

Subramaniyan M, Dani JA (2015). Dopaminergic and cholinergic learning mechanisms in nicotine addiction. Ann NY Acad Sci 1349: 46-63.

Sun J, Xu J, Cairns NJ, Perlmutter JS, Mach RH (2012). Dopamine $\mathrm{D}_{1}, \mathrm{D}_{2}, \mathrm{D}_{3}$ receptors, vesicular monoamine transporter type-2 (VMAT2) and dopamine transporter (DAT) densities in aged human brain. PLoS One 7: e49483.

Takahashi H, Fujimura Y, Hayashi M, Takano H, Kato M, Okubo Y et al (2008). Enhanced dopamine release by nicotine in cigarette smokers: a double-blind, randomized, placebo-controlled pilot study. Int J Neuropsychopharmacol 11: 413-417.

Trifilieff P, Martinez D (2014). Imaging addiction: D2 receptors and dopamine signaling in the striatum as biomarkers for impulsivity. Neuropharmacology 76 Pt B: 498-509.

Volkow ND, Wang GJ, Fowler JS, Tomasi D, Telang F (2011). Addiction: beyond dopamine reward circuitry. Proc Natl Acad Sci USA 108: 15037-15042.

Wong DF, Broussolle EP, Wand G, Villemagne V, Dannals RF, Links JM et al (1988). In vivo measurement of dopamine receptors in human brain by positron emission tomography age and sex differencesa. Ann NY Acad Sci 515: 203-214.

$\mathrm{Wu}$ Y, Carson RE (2002). Noise reduction in the simplified reference tissue model for neuroreceptor functional imaging. J Cereb Blood Flow Metab 22: 1440-1452.

Zald DH, Woodward ND, Cowan RL, Riccardi P, Ansari MS, Baldwin RM et al (2010). The interrelationship of dopamine D2-like receptor availability in striatal and extrastriatal brain regions in healthy humans: a principal component analysis of $\left[{ }^{18} \mathrm{~F}\right]$ fallypride binding. Neuroimage 51: 53-62.

Zuo Y, Mukhin AG, Garg S, Nazih R, Behm FM, Garg PK et al (2015). Sex-specific effects of cigarette mentholation on brain nicotine accumulation and smoking behavior. Neuropsychopharmacology 40: 884-892.

Supplementary Information accompanies the paper on the Neuropsychopharmacology website (http://www.nature.com/npp) 